\title{
Williams Syndrome
}

National Institute of Neurological Disorders and Stroke (NINDS)

\section{Source}

National Institute of Neurological Disorders and Stroke (NINDS). Williams Syndrome Information Page.

Williams Syndrome (WS) is a rare genetic disorder characterized by mild to moderate delays in cog nitive development or learning difficulties, a distinctive facial appearance, and a unique personality that combines over-friendliness and high levels of empathy with anxiety. The most significant medical problem associated with WS is cardiovascular disease caused by narrowed arteries. WS is also associated with elevated blood calcium levels in infancy. A random genetic mutation (deletion of a small piece of chromosome 7), rather than inheritance, most often causes the disorder. However, individuals who have WS have a 50 percent chance of passing it on if they decide to have children. The characteristic facial features of WS include puffiness around the eyes, a short nose with a broad nasal tip, wide mouth, full cheeks, full lips, and a small chin. People with WS are also likely to have a long neck, sloping shoulders, short stature, limited mobility in their joints, and curvature of the spine. Some individuals with WS have a star-like pattern in the iris of their eyes. Infants with WS are often irritable and colicky, with feeding problems that keep them from gaining weight. Chronic abdominal pain is common in adolescents and adults. By age 30 , the majority of individuals with WS have diabetes or pre-diabetes and mild to moderate sensorineural hearing loss (a form of deafness due to disturbed function of the auditory nerve). For some people, hearing loss may begin as early as late childhood. WS also is associated with a characteristic "cog nitive profile" of mental strengths and weaknesses composed of streng ths in verbal short-term memory and language, combined with severe weakness in visuospatial construction (the skills used to copy patterns, draw, or write). Within language, the strongest skills are typically in concrete, practical vocabulary, which in many cases is in the low average to average range for the general population. Abstract or conceptual-relational vocabulary is much more limited. Most older children and adults with WS speak fluently and use good grammar. More than $50 \%$ of children with WS have attention deficit disorders (ADD or ADHD), and about 50\% have specific phobias, such as a fear of loud noises. The majority of individuals with WS worry excessively. 
